\title{
New Visible and NIR Highly Photostable Fluorescent Silica Nanoparticles for Laser Scanning Imaging Applications
}

\author{
T. Ribeiro*, R. Sebastian*, A.S. Rodrigues*, F. Fernandes*, C. Baleizão* and J.P.S. Farinha*
}

*CQFM-Centro de Química-Física Molecular and IN-Institute of Nanoscience and Nanotechnology, Instituto Superior Técnico, Technical University of Lisbon, Av. Rovisco Pais, 1049-001 Lisboa, Portugal

One way to improve the photophysical and photochemical properties of NIR probes is to incorporate them in nanoparticles (NPs). The characteristics of NP-based imaging agents, such as the ability to carry large payloads of optical probes and multiple targeting groups, together with the possibility to tune their pharmacokinetics, have motivated the development of different systems for use in combined diagnostic and therapeutic (theranostic) applications [1]. The use of silica nanoparticles (SiNP) in nanomedicine, as carriers for drug delivery and imaging agents has increase remarkably in the last decade [2]. On the other hand, it is well known that the use of near infrared (NIR) light (750-1400 nm) in optical bioimaging presents several advantages over lower wavelength light (visible), namely higher tissue penetration, lower interference from autofluorescence in biological specimens, and reduced light scattering. These factors have greatly increased the interest in NIR fluorescent dyes for use in biological imaging [3]. An additional advantage of NIR dyes is that they can be used with inexpensive laser diode excitation sources in laser scanning microscopy.

In this communication we evaluate the performance of new fluorescent SiNP in laser scanning microscopy. The particles are labelled with two different perylenediimide (PDI) derivatives, one with emission in the visible region of the spectrum (PDIvis) and the other emitting in the NIR (PDInir). The PDI derivatives were covalently incorporated in the SiNPs during their synthesis by a modified Stöber method [4]. The labelled SiNP were characterized by transmission electron microscopy (TEM), scanning electron microscopy (SEM), fluorescence emission spectroscopy and laser scanning fluorescence confocal microscopy (LSFCM).

The excellent photophysical properties and stability of the fluorescently labelled SiNP encouraged us to evaluate their performance in vivo, as markers for LSFCM. The nanoparticles were internalized in HEK293 cells costained with plasma membrane (AF594-WGA) and/or nucleus (Hoechst 33258) markers. Confocal microscopy images show that both SiNP-PDInir and SiNP-PDIvis were efficiently internalized in the cells (Figure 1 and 2, respectively). The major difference between the two sets of SiNPs is that while SiNP-PDIvis is excited at $488 \mathrm{~nm}$ (argon laser) showing emission in the green region of the spectra, SiNP-PDInir can be excited at $633 \mathrm{~nm}$ (using a low power/low cost HeNe laser) with the emission observed in the NIR. The in vivo photostability of the labelled nanoparticles was compared with that of a commercial membrane marker, DiIC18(5), showing much higher photostability during laser scanning, due to the shielding effect of the silica nanoparticles.

References

1. Choi K.Y. et al., Nanoscale, 4:330, 2012.

2. Bonacchi S. et al., Chem. Int. Ed., 50:4056, 2011.

3. Hilderbrand S.A. and Weissleder R., Curr. Opin. Chem. Biol., 14:71, 2010.

4. Ribeiro T. et al., J. Phys. Chem. C, 113:18082, 2009. 


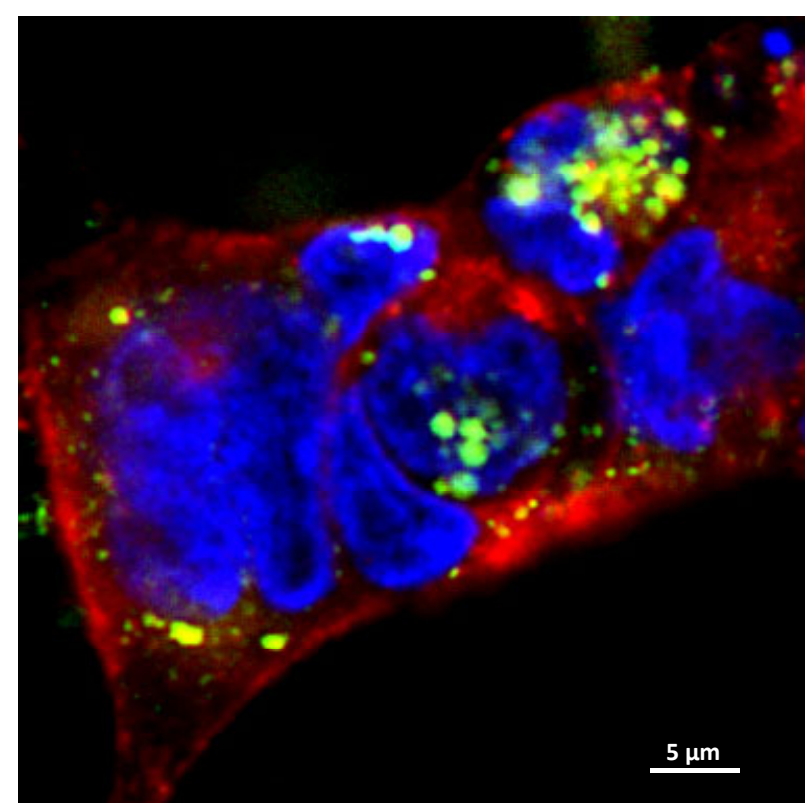

Figure 1. Confocal fluorescence image of HEK293 cells stained with AF594-WGA plasma membrane marker (red) and Hoechst 33258 nucleus marker (blue). The SiNP-PDInir (excitated at $633 \mathrm{~nm}$ ) is shown in green and appears in the cytosol.

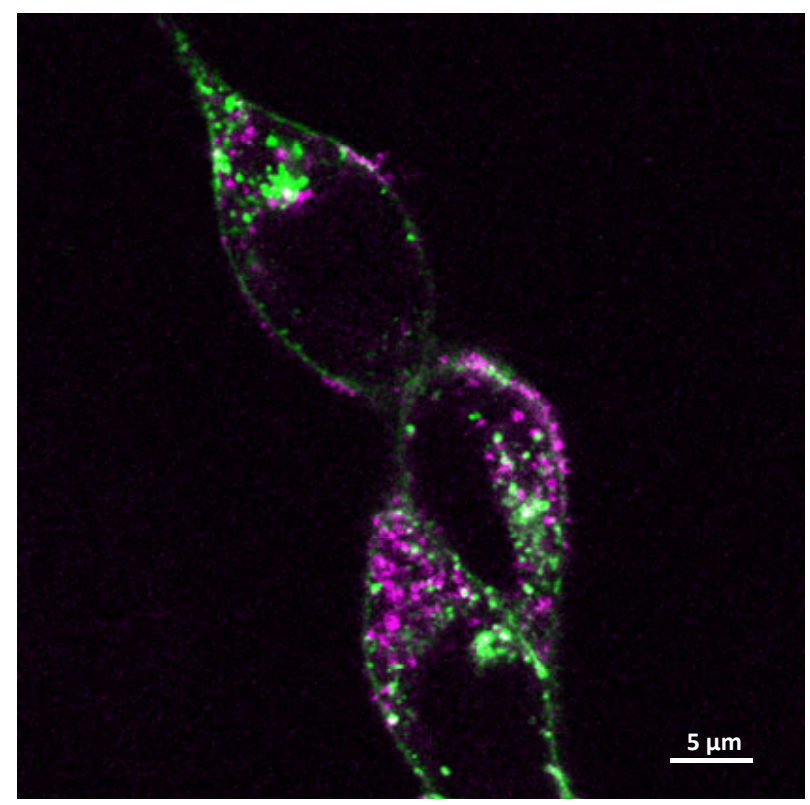

Figure 2. Confocal fluorescence image of HEK293 cells stained with AF594-WGA plasma membrane marker (green). The SiNP-PDIvis (excitated at $488 \mathrm{~nm}$ ) is shown in purple and appears in the cytosol.

This work was partially supported by Fundação para a Ciência e a Tecnologia (FCT-Portugal) and COMPETE (FEDER) within projects PTDC/CTM/101627/2008 and PEst-OE/CTM/LA0024/2011. T.R., R.S. and F.F. also thank FCT for Ph.D. (SFRH/BD/64702/2009) and Pos-Doc (SFRH/BPD/71249/2010; SFRH/BPD/64320/2009) grants. 